Supporting Information:

\title{
Near-Surface Motion and Dynamic Adhesion during Silica Microparticle Capture on a Polymer (Solvated PEG) Brush via Hydrogen Bonding
}

\author{
Surachate Kalasin and Maria M. Santore* \\ Department of Polymer Science and Engineering \\ University of Massachusetts \\ 120 Governors Drive \\ Amherst, MA 10013 \\ *Santore@mail.pse.umass.edu
}

In our paper we relied on the literature for a description of the free motion of sphere flowing near, but not engaged (though friction or bonding interactions) with, the chamber wall.

In order to describe the free motion of a sphere near a wall in a shearing flow, Goldman, Cox, and Brenner worked out an exact solution of the Stokes equations for a sphere in a viscous fluid. ${ }^{1}$ Their treatment, for a neutrally buoyant sphere near a wall in simple shearing flow, employs bipolar coordinates and provides the translational and rotational velocity of the sphere. The approach is valid for small shear Reynolds numbers (inertia is neglected) and applies for all ratios of the sphere radius to it distance from the wall.

The shear Reynolds number relevant to this problem is $R e=\frac{a^{2} s}{v}$, where $a$ is the particle radius, $v$ is the kinematic viscosity, and $S$ is the wall shear rate in the absence of the particle. For a 1-um diameter sphere in water at the largest walls shear of $500 \mathrm{~s}^{-1}$ in our experiments, $R e=\frac{\left(0.5 \times 10^{-4}\right)^{2}\left(500 \mathrm{~s}^{-1}\right)}{0.01 \frac{\mathrm{cm}^{2}}{\mathrm{~s}}}=0.000125$. All our experiments are therefore well within the regime where inertia is unimportant compared with viscous effects. Our system therefore meets the Reynolds number criteria for application of the model.

The Goldman, Cox, and Brenner 1967 publication which we employed ${ }^{1}$ is the second of a pair of co-published papers, and has been used widely by the cell rolling community to interpret cell-free models of a freely-flowing sphere in shear flow sphere flow near a wall. The first paper in the series ${ }^{2}$ solves the Stokes equations to describe the slow viscous motion of a sphere parallel to a plane wall in a quiescent fluid. The second paper builds on the first to predict the behavior the sphere in shearing flow. In developing the exact solution, Goldman et at utilize the linear superposition of the forces and torques calculated in the first paper along with additional terms arising from the shear field in the second 
paper. The exact solution of the Stokes equations for shear flow is accomplished numerically (with the details of the numerical approach described exclusively in the Goldman thesis), and incorporates the numerical results for forces and torques in quiescent solution that were tabulated in the first paper.

Table I. Numerical Results for the Dimensionless Translational Sphere Velocity, U/hS, from the Exact Solution (taken from reference 1)

\begin{tabular}{|ll|}
\hline $\mathrm{h} / \mathrm{a}$ & $\mathrm{U} / \mathrm{hS}$ \\
$\infty$ & 1.00000 \\
3.7622 & 0.009436 \\
2.3524 & 0.97780 \\
1.5431 & 0.92368 \\
1.1276 & 0.76692 \\
1.0453 & 0.65375 \\
1.005004 & 0.47861 \\
1.0032002 & 0.45921 \\
\hline
\end{tabular}

Here $\mathrm{h}$ is the distance from the sphere center to the wall, a is the particle radius. $\mathrm{U}$ is the translational velocity, and $\mathrm{S}$ is the wall shear rate.

The tabulated results of the exact solution, reproduced here in Table I from the second paper, were originally presented along with two asymptotic solutions: (A) a method of reflections approximation when the sphere is far from the wall and (B) a lubrication-theory treatment that applies in the limit when the sphere is close to the wall compared with its radius. The authors state, in their second paper that the tabulated results, along with the lubrication theory results furnish a complete description of the sphere's motion, but for completely we include both limiting forms here:

From the method of reflections:

$$
\frac{U}{h S}=1-\frac{5}{16}\left(\frac{a}{h}\right)^{3}
$$

Lubrication treatment $(\delta=h-a)$ :

$$
\frac{U}{h S} \sim \frac{0.7431}{0.6376-0.200 \ln \left(\frac{\delta}{a}\right)}
$$


As shown in the second Goldman paper, in their respective limits, equations 1 and 2 match the exact solutions to 4 decimals. However, as we need only two significant figures on $\mathrm{U} / \mathrm{hS}$, it is useful to note that equation 1 matches table 1 above $\mathrm{h} / \mathrm{a} \sim 1.5$, and equation 2 matches table 1 below $\mathrm{h} / \mathrm{a} \sim 1.044$. A spline fit to the table was employed at intermediate values when converting intermediate velocity values to particle-surface separations.

(1) Goldman, A. J.; Cox, R. G.; Brenner, H. Slow Viscous Motion of a Sphere Parallel to a Plane Wall .2. Couette Flow. Chem. Eng. Sci. 1967, 22, 653.

(2) Goldman, A. J.; Cox, R. G.; Brenner, H. Slow Viscous Motion of a Sphere Parallel to a Plane Wall .I. Motion through a Quiescent Fluid. Chem. Eng. Sci. 1967, 22, 637. 